\title{
Peripartum amniotic fluid embolism with disseminated intravascular coagulation during ongoing coronavirus disease pandemic - A case report
}

\author{
Sangeeta Khanna ${ }^{1}$, G V Krishna Prasad ${ }^{2}$, Anish Kumar Vishal ${ }^{3}$, Dinesh Bhasin ${ }^{4}$, Sravan Kumar Reddy Kasa ${ }^{5}$ \\ From ${ }^{1}$ Head, Department of Anesthesiology, ${ }^{2}$ Classified Specialist, ${ }^{5}$ Resident, Department of Anaes, ${ }^{3}$ Graded Specialist, ${ }^{4}$ Classified Specialist, \\ Department of $O B G$, Military Hospital, Kirkee, Maharashtra, India
}

\begin{abstract}
Amniotic fluid embolism (AFE) is a relatively rare but potentially catastrophic obstetric emergency. Early studies revealed mortality rates as high as $61-86 \%$, but more recent estimates suggest a case fatality of $13-26 \%$ with adverse neonatal outcomes. The decrease in mortality can be because of the high index of suspicion and aggressive management practices. We report a case of AFE which manifested atypically with severe back pain and respiratory distress in operation theater before anesthesia induction for elective cesarean (lower segment cesarean section).
\end{abstract}

Key words: Amniotic fluid embolism, Back pain, Coronavirus disease-19, Prepartum onset

A

mniotic fluid embolism (AFE) is a relatively rare but potentially catastrophic obstetric emergency. Early studies revealed mortality rates as high as $61-86 \%$, but more recent studies suggest a case fatality of $13-26 \%$ with adverse neonatal outcomes [1]. The incidence ranges from $1: 8000$ to $1: 80,000$ pregnancies [2]. Despite the reduction in mortality, it still accounts for a significant number of maternal deaths in the USA, the UK, and some other countries. Exact figures from India could not be found. There are no proven risk factors though age more than 35 years, cesarean section, placenta previa, and multiple pregnancies have been highlighted [3]. There are no proven mechanisms either though two theories have been propounded. Initially thought to be due to mechanical obstruction caused by fetal amniotic fluid contents, now it is proposed to be due to an anaphylactic reaction to fetal content in maternal circulation [2]. Despite advances in diagnostics, AFE still remains a clinical diagnosis and no specific tests are recommended, however, a multidisciplinary approach is important for favorable outcomes [4].

We present a case of AFE which manifested atypically with severe back pain and respiratory distress in OT before anesthesia induction for elective lower segment cesarean section (LSCS) which fortunately responded favorably to aggressive management.

\section{Access this article online}

Received - 27 July 2020

Initial Review - 12 August 2020

Accepted - 13 August 2020

DOI: $10.32677 /$ IJCR.2020.v06.i08.013

\section{CASE REPORT}

A 27-year-old second gravid (post-LSCS) with 38 weeks of gestation, booked case, was posted for elective cesarean section. The patient was accepted in American Society of Anesthesiologists-II for pregnancy and the plan of anesthesia was sub-arachnoid block. A self-declaration for coronavirus disease (COVID)-19 before surgery was not contributory and she also underwent rapid polymerase chain reaction test for COVID-19 as per existing guidelines.

On the day of surgery, just after transferring her on the operation table, she had a sudden, excruciating episode of back pain along with cough. The vital signs recorded at that time showed heart rate 126/min, $\mathrm{SpO}_{2} 98 \%$, and blood pressure of $120 / 90 \mathrm{mmHg}$.

Electrocardiography showed normal sinus rhythm. Fetal heart rate (FHR) monitored by Doppler suggested severe bradycardia with $\mathrm{FHR}<80 / \mathrm{min}$. A quick spinal anesthetic was administered (as the patient positioning had already been done) with $2.20 \mathrm{ml}$ of $0.5 \%$ hyperbaric bupivacaine between the L 3 and L 4 spines in the left lateral decubitus position. The quick cold sense check was performed with an alcohol swab to verify the sensory block level to the fourth thoracic spinal segment. A limp male baby (APGAR score of 02 ) was delivered and was successfully resuscitated by attending pediatrician.

After delivering the baby, the patient suddenly developed tachycardia $150 \mathrm{bpm}$, hypotension $50 / 28 \mathrm{~mm}$ of $\mathrm{Hg}$, and tachypnea 38/min with wheeze on auscultation. She complained

Correspondence to: Dr. G V Krishna Prasad, Department of Anaesthesiology, Military Hospital, Kirkee - 411 020, Maharashtra, India. E-mail: drkaypee99@ yahoo.com

(c) 2020 The Author(s). This open access article is distributed under a Creative Commons Attribution (CC-BY) 4.0 license. 
of severe dyspnea and continued to have a dry cough. Because of the mode of presentation and differential diagnosis of an anaphylactic reaction (antibiotics), pulmonary thromboembolism and AFE were kept in mind.

Fluid resuscitation was started by establishing two more $18 \mathrm{G}$ peripheral intravenous lines. Hypotension was managed with bolus doses of Inj. mephentermine IV initially in $3 \mathrm{mg}$ aliquots and Inj. adrenaline $0.5 \mathrm{mg} \mathrm{IM}$, and later on, the infusion of noradrenaline and dopamine was started. To relieve bronchospasm, Inj. hydrocortisone $100 \mathrm{mg}$ repeated thrice along with multidose inhaler levosalbutamol puffs. The patient was assisted with bag and mask ventilation with $\mathrm{O}_{2}$ at $8-10 \mathrm{l} / \mathrm{min}$ till she developed good spontaneous breathing, keeping difficult intubation cart and drugs standby for quickly securing the airway if required. Verbal communication was maintained with the patient throughout. After the above measures for $20 \mathrm{~min}$, vitals were as follows: Blood pressure $-90 / 50 \mathrm{mmHg}$, pulse rate $-130 / \mathrm{min}$, respiratory rate $-25 \mathrm{bpm}$, and $\mathrm{SpO}_{2}-94 \%$ with non-rebreather mask. Soon after closing the incision, the patient found to be bleeding from multiple sites (incision, intravenous cannulation, and fresh bleeding from the vagina) indicating the ongoing coagulopathy. Arterial blood gas (ABG) readings were $\mathrm{pH} 7.29, \mathrm{PCo}_{2} 25, \mathrm{HCO}_{3}$ 11.6, $\mathrm{BE}-15)$, and lactate -3.52 .

In view of the ongoing blood loss despite adequate support, emergency hysterectomy was done to avoid further blood loss because of uterine atony and disseminated intravascular coagulation (DIC). Intraoperatively, ongoing coagulopathy managed with whole blood, 2 fresh frozen plasma, and packed red blood cell. Blood samples sent for laboratory analysis revealed raised prothrombin time, activated partial thromboplastin time, international normalized ratio, D-dimer, and low fibrinogen levels. The patient was shifted to intensive care unit (ICU) after all vital parameters were relatively better.

The patient was managed in ICU for 12 days. The first 3 days her hemodynamics were unstable and ongoing blood loss amounting to $3.5 \mathrm{~L}$. She was managed with massive transfusion protocol (a total of 43 units of blood and blood products were transfused) for ongoing DIC. Along with severe hypokalemia, she also developed multiorgan involvement with deranged renal and liver parameters and tachypnea with bilateral crackles in the lungs. Her hemoglobin was on the decline despite adequate transfusion and dry drains after the $4^{\text {th }}$ day postoperatively. She was managed with empirical broad-spectrum antibiotics as the culture and sensitivity reports of blood and urine were negative. Investigations specific for AFE were sent after $12 \mathrm{~h}$ (was not available locally) of surgery were inconclusive. Monitoring was done with regular ABGs to identify acute respiratory distress syndrome or transfusion-related acute lung injury which is always a possibility in massive transfusions. Peripheral blood smear sent for evaluation of persistent anemia after multiple blood transfusions showed the picture of hemolytic uremic syndrome which was managed conservatively. She was shifted to the ward after starting on oral feeds and subsequently discharged on the $14^{\text {th }}$ day.

\section{DISCUSSION}

AFE remains a diagnosis of exclusion and should always be considered early in the course of clinical management of any obstetric emergency involving cardiovascular collapse. This patient developed severe back pain with cough followed by almost immediate fetal distress. Anaphylaxis was our initial concern because of the signs and symptoms such as a wheeze and cardiovascular collapse. Initially, the patient was managed with fluid resuscitation and adrenaline intramuscular; but after the onset of DIC, a retrograde analysis made us inclined toward AFE among all the differential diagnoses available.

The classic manifestations of AFE include sudden hypoxia, severe hypotension, and DIC. The chances of AFE occur during labor, during cesarean section, and after delivery in the immediate postpartum are $70 \%, 11 \%$, and $9 \%$, respectively [5]. Our case presented with prodromal symptoms of acute back pain and cough; during the onset of these prodromal symptoms, she was neither in labor nor she has undergone cesarean section which is very rare.

AFE is a diagnosis of exclusion and it is impossible to rule out all other differentials when an emergency is going on. Many biomarkers are presently under study, and some are available commercially [6]. Their clinical use is doubtful and not recommended routinely. Supportive therapy as indicated by clinical circumstance always remains the most important intervention and should supersede diagnostic studies. Attempts at obtaining blood or fluid samples for unvalidated diagnostic purposes should never interfere with resuscitation. In this patient, we solely treated her based on basic tests of coagulation and clinical response.

Oxygenation, circulatory support, and correction of coagulopathy continue to be the mainstays of therapy. Hypoxia and respiratory distress are to be managed with $100 \%$ oxygen. Although early intubation and mechanical ventilation are to be considered [7], the type of respiratory support will be decided based on the severity of the case [8]. Although we were ready for intubation in this patient, we avoided it by providing respiratory support by the bag and mask for about 20 min until the patient regained her full spontaneous effort.

Hemodynamic instability needs to be treated with careful volume expansion, vasopressors, and inotropes. Management priorities will change from case to case and availability of equipment as depicted in the flowchart (Fig. 1) [9]. Invasive arterial pressure monitoring and central venous line placement will be helpful but one should not compromise and delay the lifesupporting treatment while doing these procedures. In our case, we established a central line after shifting the patient to ICU to monitor central venous pressure and used non-invasive blood pressure for monitoring. Transfusion of blood products forms the cornerstone of treating coagulopathy. Massive blood transfusion protocol needs to be initiated as soon as AFE is suspected. Blood and component therapy should be guided by clinical presentation and coagulation status. Close communication with blood banks is paramount because large quantities of blood and components required. The role of heparin and factor VIIa is controversial and should be used with caution depending on the clinical scenario. 


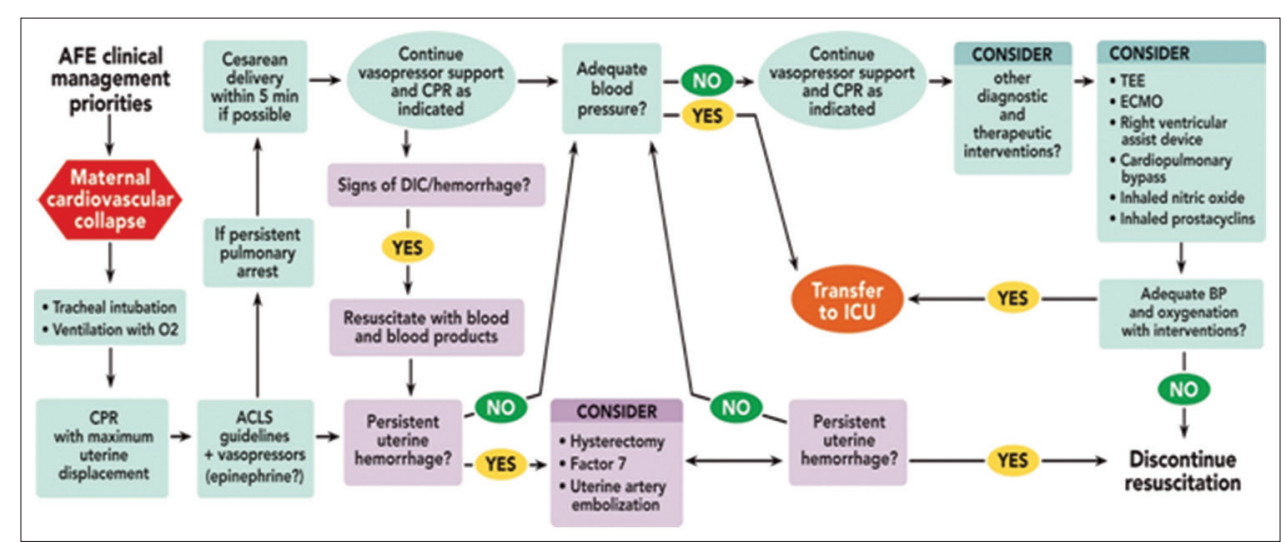

Figure 1: Flowchart depicting clinical management priorities for amniotic fluid embolism [9]. ACLS: Advanced cardiac life support; AFE: Amniotic fluid embolism; BP: Blood pressure; CPR: Cardiopulmonary resuscitation; DIC: Disseminated intravascular coagulation; ECMO: Extracorporeal membrane oxygenation; ICU: Intensive care unit; TEE: Transesophageal echocardiography

\section{CONCLUSION}

AFE is a near-fatal condition seen in the obstetric population exclusively. It is usually a diagnosis of exclusion, as diagnostic modalities such as computed tomography, angiography, and other blood biomarkers might not be available or feasible for the confirmation of the diagnosis in all centers like our peripheral hospital. A high degree of suspicion and aggressive resuscitation measures and supportive care could save both parturient and the fetus in both the operating room and ICU.

\section{REFERENCES}

1. Abenhaim HA, Azoulay L, Kramer MS, Leduc L. Incidence and risk factors of amniotic fluid embolisms: A population-based study on 3 million births in the United States. Am J Obstet Gynecol 2008;199:49-8.

2. Dedhia JD, Mushambi MC. Amniotic fluid Embolism. Contin Educ Anesth Crit Care Pain 2007;7:152-6.

3. Saade G, Hankins GD, Clark S. Amniotic fluid embolism: Diagnosis and management. Am J Obstet Gynecol 2016;215:B16-24.

4. Rath WH, Hoffer S, Sinicina I, Arztebl D. Amniotic fluid embolism: An interdisciplinary challenge: Epidemiology, diagnosis and treatment. Dtsch Arztebl Int 2014;111:126-32.

5. Pantaleo G, Luigi N, Federica T, Paola S, Margherita N, Tahir M. Amniotic fluid embolism: Review. Curr Pharm Biotechnol 2014;14:1163-7.

6. Stawicki SP, Papadimos TJ. Challenges in managing amniotic fluid embolism: An up-to-date perspective on diagnostic testing with focus on novel biomarkers and avenues for future research. Curr Pharm Biotechnol 2014; 14:1168-78.

7. Gist RS, Stafford IP, Leibowitz AB, Beilin Y. Amniotic fluid embolism. Anesth Analg 2009;108:1599-602.

8. McDonnell NJ, Percival V, Paech MJ. Amniotic fluid embolism: A leading cause of maternal death yet still a medical conundrum. Int J Obstet Anesth 2013;22:329-36.

9. Laura S, Rogers RP. Case scenario: Amniotic fluid embolism. Anesthesiology 2012;116:186-92.

Funding: None; Conflicts of Interest: None Stated.

How to cite this article: Khanna S, Prasad GV, Vishal AK, Bhasin D, Kasa SK. Peripartum amniotic fluid embolism with disseminated intravascular coagulation during ongoing coronavirus disease pandemic - A case report. Indian J Case Reports. 2020;6(8):458-460. 